\title{
1D Nano-Ultrasonic Scan with 1-Nanometer Spatial Resolution
}

\author{
Kung-Hsuan Lin and Cheng-Ta Yu \\ Graduate Institute of Electro-Optical Engineering, National Taiwan University, Taipei, 10617, Taiwan \\ Phone :886-2-23659703, FAX :886-2-23677467,E-mail :f90941009@ntu.edu.tw \\ Chang-Chi Pan and Jen-Inn Chyi \\ Optical Sciences Center, National Central University, Chung-Li 32054, Taiwan \\ Sheng-Wen Huang and Pai-Chi Li \\ Department of Electrical Engineering, National Taiwan University, Taipei 10617, Taiwan \\ Chi-Kuang Sun \\ Department of Electrical Engineering and Graduate Institute of Electro-Optical Engineering, National Taiwan University, Taipei, 10617, \\ Taiwan
}

\begin{abstract}
Piezoelectric quantum wells can be treated as opto-acoustic transducers to generate and detect acoustic waves with nanometer wavelengths. This opto-acoustic transducer was utilized for $1 \mathrm{D}$ ultrasonic scan with 1 nanometer demonstrated resolution. C2005 Optical Society of America

OCIS codes: 320.7100 Ultrafast measurements; 320.7130 Ultrafast processes in condensed matter, including semiconductors
\end{abstract}

\section{Introduction}

$\mathrm{THz}$ coherent longitudinal-acoustic (LA) phonon oscillation generated in piezoelectric multiple quantum wells was recently experimentally demonstrated [1]. By generating the coherent LA phonons only in the quantum wells, these acoustic phonon oscillations can be treated as an acoustic wave with wavelength of several nanometers, which is determined by the period of quantum wells. Because the acoustic wave is primarily induced through the piezoelectric effect [1], this structure is actually an optical piezoelectric transducer (OPT) for the generation of nanoacoustic waves (NAWs). Compared with a typical electrical transducer, the main difference is that the variation of electric field in the multiple-quantum-well structure is induced by femtosecond laser pulses instead of electric pulses.

In this paper, we demonstrate a 1D nano-ultrasonic scan measurement by using the optically generated NAWs. By launching a NAW pulse and femtosecond-time-resolving the intensity and phase of returned acoustic echo signals, the distance of the studied interface can be accurately measured with a resolution down to 1 nanometer. This technique provides a non-destructive method to measure the thickness of a nano-thin film on the surface or embedded in the sample with a nanometer resolution.

\section{Experimental Setup and Results}

To design an OPT for 1D ultrasonic scan, NAW with fewer oscillation cycles, thus with a shorter acoustic pulsewidth, is required. We used piezoelectric InGaN/GaN MQW as the OPT for the generation of NAWs. The OPT with a 3-period $29 \AA / 130 \AA \mathrm{In}_{0.23} \mathrm{Ga}_{0.77} \mathrm{~N} / \mathrm{GaN}$ QW structure was grown by MOCVD on top of a GaN buffer layer with a thickness of more than $2 \mu \mathrm{m}$ on a c-plane sapphire substrate [2]. Then a $\mathrm{GaN}$ cap layer was grown on top of the OPT as the studied propagating layer for the ultrasonic thickness measurement. The nitride layers are all with a wurzite structure. The thickness of the cap layer is estimated to be $\sim 80 \mathrm{~nm}$ according to the growth rate.

Fig. 1 shows the result of the transient optical transmission measurement performed on the 3-period $29 \AA / 130 \AA$ InGaN/GaN OPT. The oscillating period is $2 \mathrm{ps}$, corresponding to an acoustic frequency of $0.5 \mathrm{THz}$ (acoustic wavelength of $159 \AA$ ). Around zero-time-delay while the strain pulse was generated in the quantum well region of the OPT, an increase in the optical transmission can be observed due to the carrier bandfilling effect. While the carrier-induced periodic strain pulses traveled out of the wells where they are generated and crossed the neighbored quantum wells, two extra transmission oscillation peaks could be observed after zero time-delay. One of the generated NAW left the OPT and traveled in the sample-growth-direction toward the sample surface, reflected at the sample surface, and echoed back into the OPT starting at $\sim 20 \mathrm{ps}$. Note that the echoed strain pulses decreased the 


\section{QTuK5}

background optical transmission because the sign of the echoed strain was reversed at the GaN/air interface, which is a free end. While the echoed NAW with three-cycles traveling through the QW region, it results in five optical transmission dips on the background signal. The transient central position of the five dips corresponds to the moment when the three-cycle NAW was located in three-quamtum-wells.

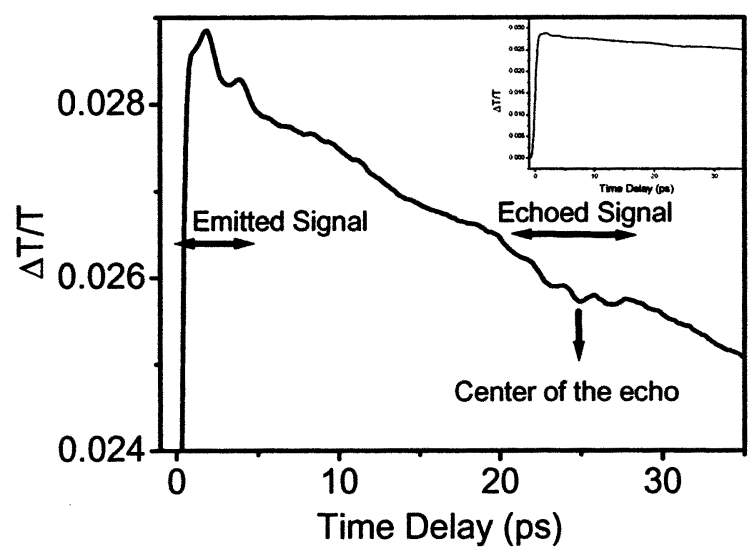

Fig. 1. Measured optical transient transmission changes versus probe delay for a 3-period $29 \AA / 130 \AA$ InGaN/GaN OPT. The complete trace is shown in the inset.

\section{Signal Processing}

To filter the background signal and accurately resolve the peak position of the echo signal, we have successfully developed a filter technique based on the ultrasonic imaging principle. Fig. 2 shows the filtered signal in a solid line while showing the envelope in a dashed line. We found that the peak position of the echo signal (the third oscillation peak) is around $25.4 \mathrm{ps}$ with respect to the excitation time. Note that the peak position of 25.4 ps does not represent the traveling time of NAW through the GaN cap layer. It represents the required time that the generated NAW traveled into the cap layer, reflected from the cap/air interface, then went back to match the period of quantum wells in OPT.

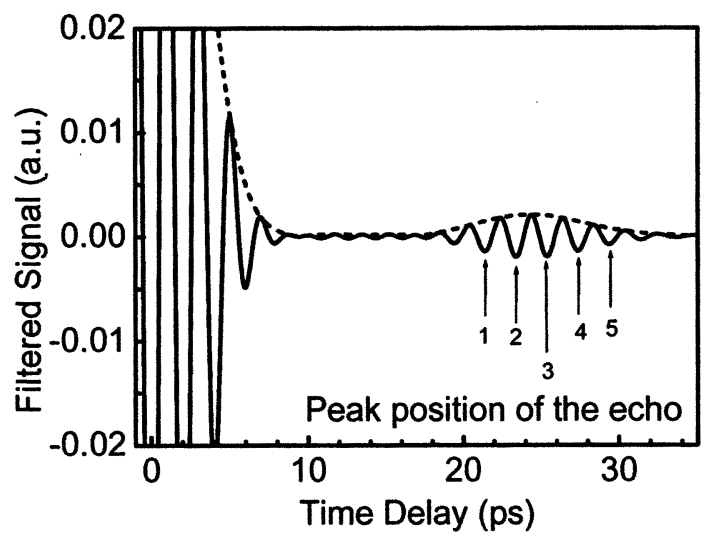

Fig. 2. The trace in Fig. 1 after filtering (solid line). Its envelope is shown as dashed line. Five echoed peaks are clearly observable. Two more side peaks are caused by filter artifact.

To calculate the accurate thickness of the GaN cap layer grown on the OPT, the time that NAW travels in the cap layer should be obtained by subtraction of the time spent in the OPT. In the case of 3-period QW OPT, three strain pulses were generated in the three InGaN well layers. We take the strain pulse generated in the first InGaN strained layer below the $\mathrm{GaN}$ cap layer (called strain pulse A) as an example to calculate the traveling time in the $\mathrm{GaN}$ cap layer. While the strain pulse A came back from the GaN/air interface, it entered the first InGaN strained 


\section{QTuK5}

layer below the GaN cap layer and resulted in the first dip signal of the echo signal as shown in Fig. 2. While the strain pulse A crossed the second well and arrived at the third well, it resulted in the third dip of the echo signal, which is the peak position. Therefore, the traveling time in the GaN cap layer is that the peak position of the echo signal subtracts the time in the OPT with two GaN barriers and three InGaN wells. With an oscillation period of $2 \mathrm{ps}$, the traveling time in the OPT is $4.4 \mathrm{ps}$, resulting in $21.0 \mathrm{ps}$ traveling time in the GaN cap layer. Assuming the sound velocity of GaN along c-axis is $8020 \mathrm{~m} / \mathrm{s}$ [3], the thickness of the GaN cap layer is thus estimated to be $\sim 84 \mathrm{~nm}$.

\section{Discussions}

Since the time resolution of the optical detection is determined by the optical probe pulse width, not only high speed but also high spatial resolution can thus be achieved. For example, with an optical probe-pulsewidth of $100 \mathrm{fs}$ and an acoustic velocity of $8000 \mathrm{~m} / \mathrm{s}$, the spatial resolution is better than $0.8 \mathrm{~nm}$ (with a fitting procedure, a time resolution better than pulsewidth can be easily achieved as in our case). As for the detection of thin multi-layers, the actual spatial resolution is also restricted by the acoustic wavelength and the acoustic pulsewidth. By resolving the envelope of the reflected acoustic echo, NAW can easily provide a spatial resolution down to several nanometers. By resolving the phase information (as exampled in Figure 2) and the interference between different echoes due to multi-layers, a spatial resolution down to several angstroms is plausible.

For a single layer thickness measurement without multiple reflections, taking advantage of the phase information, a resolution better than $20^{\circ}(\lambda / 16)$, corresponding to $<1 \mathrm{~nm}$ resolution in this specific case is achievable. However the uncertainty of the acoustic velocity also results in the measurement errors. For example, a $100 \mathrm{~m} / \mathrm{s}$ uncertainty will give rise to a $1.25 \%$ inaccuracy for the determination of the $\mathrm{GaN}$ layer thickness (in this case, $\sim 1 \mathrm{~nm}$ ). Detailed discussions about the technologies of $1 \mathrm{D}$ nano-ultrasonic scan will be presented in the conference.

\section{Acknowledgement}

This work is sponsored by National Science Council of TAIWAN.

\section{References}

[1] G.-W. Chern, C.-K. Sun, G. D. Sanders, and C. J. Stanton, "Generation of Coherent Acoustic Phonons in Nitride-Based Semiconductor Nanostructures," Top. Appl. Phys. 92,339 (2004).

[2] C.-M. Lee, C.-C. Chuo, I.-L. Chen, J.-C. Chang, and J.-I. Chyi, "High-brightness inverted InGaN-GaN multiple-quantum-well light-emitting diodes without a transparent conductive layer," IEEE Electron. Dev. Lett. 24, 156 (2003).

[3] C. Deger, E. Born, H. Angerer, O. Ambacher, M. Stutzmann, J. Hornsteiner, E. Riha, and G. Fischerauer, "Sound velocity of AlxGa1-xN thin films obtained by surface acoustic-wave measurements," Appl. Phys. Lett. 72, 2400 (1998). 